\title{
Novel method for the detection and quantification of malignant cells in the CSF of patients with leptomeningeal metastasis of lung cancer
}

\author{
CHUNHUA MA*, YUAN LV*, RONG JIANG, JINDUO LI, BIN WANG and LIWEI SUN \\ Tianjin Key Laboratory of Cerebral Vascular and Neurodegenerative Disease, Department of Intervention, \\ Tianjin Huanhu Hospital, Tianjin 300060, P.R. China
}

Received January 15, 2015; Accepted October 23, 2015

DOI: $10.3892 / \mathrm{ol} .2015 .3971$

\begin{abstract}
The aim of the present study was to discuss a novel method for the detection of malignant tumor cells in cerebrospinal fluid (CSF), by observing tumor marker-immunostaining fluorescence in situ hybridization (TM-iFISH) enrichment and by counting CSF malignant tumor cells in patients with lung cancer leptomeningeal metastasis (LM). A total of 10 CSF samples were collected from 6 patients that presented with lung cancer LM. For each patient, $20 \mathrm{ml}$ CSF was obtained through a lumbar puncture, of which $7.5 \mathrm{ml}$ was used to count the number of malignant tumor cells in the CSF using TM-iFISH enrichment. Cytological and biochemical examinations were conducted on the remaining $10 \mathrm{ml}$ and $2.5 \mathrm{ml}$ CSF, respectively. The $10 \mathrm{CSF}$ samples were successfully analyzed by TM-iFISH, and the tumor cell count range was 3-1,823 cells/7.5 $\mathrm{ml} \mathrm{CSF}$ in 7 of the samples detected. There were no tumor cells detected in the remaining 3 samples. Tumor cells were revealed in 3 of the samples through the CSF cytological examinations, and albumin protein levels were indicated to be greater than the normal range (normal range, 0.15-0.45 g/l), in 9 of the samples using CSF biochemical examinations. Additionally, TM-iFISH was performed again to count the CSF malignant tumor cells in 3 of the patients following intrathecal injection of chemotherapy (methotrexate $10 \mathrm{mg}$ and dexamethasone $5 \mathrm{mg}$ ). The results indicated that the malignant tumor cell count of 2 of the patients had decreased in comparison to the pre-treatment cell count. As it is capable of
\end{abstract}

Correspondence to: Dr Rong Jiang, Tianjin Key Laboratory of Cerebral Vascular and Neurodegenerative Disease, Department of Intervention, Tianjin Huanhu Hospital, 122 Qixiangtai Road, Tianjin 300060, P.R. China

E-mail: jiangrong1989@sina.com

${ }^{*}$ Contributed equally

Key words: carcinoma, cerebrospinal fluid, leptomeningeal metastasis, neoplasm circulating cells, non-small-cell lung, non-small lung cancer enriching and counting CSF malignant tumor cells in patients with lung cancer LM, TM-iFISH may be an effective method to diagnose lung cancer LM and to evaluate its efficacy.

\section{Introduction}

Leptomeningeal metastasis (LM) is a central nervous system metastatic carcinoma caused by the diffuse dissemination or focal infiltration of cancer cells into the meninges and spinal subarachnoid space (1). LM occurs in primary lesions and frequently occurs secondary to leukemia, lymphoma and lung and breast cancer (1). The median survival time of patients with untreated LM is 4-6 weeks, while patients provided with comprehensive treatment may survive for 3-5 months (2). At present, the early diagnosis of LM is, to a certain extent, a medical problem. The diagnostic methods mainly include the examination of central nervous system symptoms, cerebrospinal fluid (CSF) cytological examinations and brain enhanced magnetic resonance imaging (MRI) scans. However, $>90 \%$ patients with LM do not show symptoms of the disease in the central nervous system (3). The rate of patients that test LM-negative at the first CSF cytological examination is $\leq 45 \%$, yet the rate of patients that test LM-positive at the second examination is $\leq 80 \%$ (4). Brain enhanced MRI scans may reach $100 \%$ specificity, yet $65 \%$ of scans falsely assess patients as LM-negative and $10 \%$ of scans falsely assess patients as LM-positive (5). Therefore, finding a more sensitive method for the detection of potential LM is required.

Previous studies demonstrated that circulating tumor cells (CTCs), particularly deciduous solid tumor cells, in the blood are associated with tumor metastasis, drug resistance (6), prognosis and recurrence $(7,8)$. CTCs may also associate with non-hematogenous epithelial cells, and the majority of CTCs express cytokeratin $(\mathrm{CK})$ in epithelial cells. In addition, certain chromosomes, including chromosome 8 , also exhibit abnormal manifestations, such as being non-diploid, haploid or polyploid (9). Tumor marker-immunostaining fluorescence in situ hybridization (TM-iFISH) may effectively identify and count various non-hematogenous tumor epithelial cells in biological fluid samples using enrichment and analysis techniques (9). In total, 6 patients, who were previously diagnosed with lung cancer LM using cytology or imaging examinations at the Department 
of Intervention of Tianjin Huanhu Hospital (Tianjin, China), were selected for the present study. The $10 \mathrm{CSF}$ samples obtained were examined for malignant tumor cells using TM-iFISH, in order to investigate the suitability of the novel method for the assessment of the development of CSF malignant tumor cells.

\section{Materials and methods}

Inclusion criteria. The 6 patients with LM of non-small cell lung cancer (NSCLC) that were treated in Tianjin Huanhu Hospital (Tianjin, China)were enrolled in the present study. All patients met the following standards: i) NSCLC, diagnosed by histology or cytology; ii) LM, diagnosed by CSF cytology or imageology; iii) normal clotting time (normal value, $12-14 \mathrm{sec}$ ), and platelet count (normal range, 100-300 x 10 $/ 1$ ); iv) controllable intracranial hypertension following treatment with dehydrated drugs $(250 \mathrm{ml} 20 \%$ mannitol intravenously every $8 \mathrm{~h}$ ); v) tolerance to lumbar puncture for the collection of CSF; vi) no meningeal lesions, including intracranial meningioma, ependymoma and spinal meningioma; and vii) a signed informed consent form. The patients received intrathecal injection chemotherapy of $10 \mathrm{mg}$ methotrexate and $5 \mathrm{mg}$ dexamethasone, once a week. The regimens of intravenous chemotherapy was pemetrexed , intravenous drip, $500 \mathrm{mg} /$ $\mathrm{m}^{2}$, once every 21 days. The study was approved by the ethics committee of Tianjin HuanHu Hospital Ethics committee.

Detection methods. In total, $20 \mathrm{ml} \mathrm{CSF}$ was drawn from all patients by lumber puncture, of which $7.5 \mathrm{ml}$ was stored in a TM-iFISH detection tube at room temperature. TM-iFISH was used to detect CTCs within 3 days of CSF extraction. The specific method was split into two parts: Cell enrichment and cell analysis.

\section{TM-iFISH}

Cell enrichment. The cerebrospinal fluid (CSF) was treated with immunomagnetic beads coated with lymphocyte common antigen (CD45) antibody (Cytelligen, Inc., San Diego, CA, USA), in order to remove CD45-positive leukocytes. The CSF was subsequently centrifuged for $5 \mathrm{~min}$ at $110 \mathrm{x} \mathrm{g}$. The supernatant was discarded and the cell pellet was diluted with saline to yield a $100 \mu 1$ cell suspension.

Cell analysis. The cells were counted and nucleic acids were detected as follows. The $100 \mu 1$ cell suspension solution was first fixed. Then, centromeric probe 8 (CEP8) was used to detect certain factors for TM-iFISH, including the numbers of chromosome 8, the anti-cytokeratin 18 (CK-18) antibody, which captures cells that are derived from the epithelium, and the CD45 antibody, which demonstrates that the captured cells are non-leukocytes. The cells were stained with 4',6-diamidino-2-phenylindole (DAPI), as the cells were karyocytes. The number of cells was counted using an Olympus BX53 fluorescence microscope (Olympus Corporation, Tokyo, Japan). The count was repeated 5 times, and the mean was selected as the final value for each patient. The remaining 10 and $2.5 \mathrm{ml} \mathrm{CSF}$ were used to conduct cytological and biochemical examinations, respectively, subsequent to sample collection.

Judgment standards of CTCs. The CTCs did not express the surface markers of hematogenous cells, such as CD45.

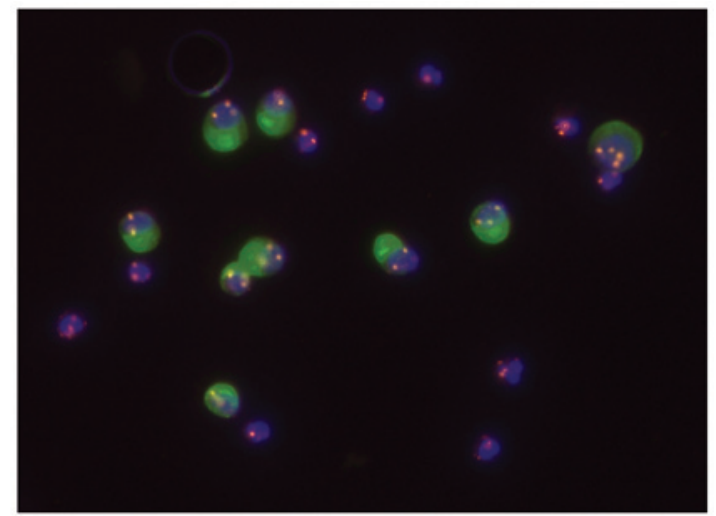

Figure 1. Tumor cells were visible under fluorescence microscopy (magnification, $\mathrm{x} 400$ ). The nucleus was stained with 4',6-diamidino-2-phenylindole (blue). Lymphocyte common antigen was expressed in the cells (red circle around the nucleus), and cytokeratin-18 was also highly expressed (green). The number of chromosomes was diploid or triploid, as exhibited by chromosome enumeration probe 8 during fluorescence in situ hybridization detection.

Under the fluorescence microscopy, non-hematogenous naked nuclear cells, so named due to the lack of a red halo around the cell nucleus, that did not express CD45 were observed under red channels. The CEP8 iFISH signal was examined under an orange channel, and it was revealed that chromosome 8 was polyploid in the majority of the CTCs. The expression of CK-18 was then observed under green channels, with green fluorescence conferring CK-18 expression. DAPI-stained cells were observed under blue channels, with blue fluorescence conferring expression. Therefore, tumor cells originating from non-hematogenous epithelial cells were evident when the detection markers of CTCs in the CSF manifested as DAPI ${ }^{+}$ $\mathrm{CD}^{-} 5^{-}, \mathrm{CK} 18^{+}$or $\mathrm{CK} 18^{-}$and $\mathrm{CEP} 8^{+}$. The chromosomes of the tumor cells may be haploid, diploid or polyploid. However, the CTCs were evidently hematogenous leukocytes when the markers $\mathrm{DAPI}^{+}, \mathrm{CD}^{2} 5^{+}, \mathrm{CK} 18^{-}$and $\mathrm{CEP}^{+}$were presented, of which the overwhelming majority of chromosomes were diploid.

\section{Results}

Clinical characteristics of 6 patients. The 6 patients with LM of lung cancer that met the inclusion criteria were all female, with a median age of 57.5 (range, 46-64 years). The primary tumor pathological type was lung adenocarcinoma. Among the 6 patients, there were 4 patients with an epidermal growth factor receptor (EGFR) gene mutation, but without a Kirsten rat sarcoma viral oncogene homolog (K-Ras) gene mutation, 1 patient without the EGFR and K-Ras gene mutations and 1 patient with unknown gene mutations. There were 5 patients that developed brain parenchyma metastasis complications. The major clinical manifestations included a persistent headache (5 patients), cognitive disorder (3 patients), dysopia ( 2 patients), deafness ( 2 patients) and cauda equina syndrome (2 patients) (Table I).

Detection of cells in 10 CSF samples. Among the 6 patients, TM-iFISH successfully analyzed in a total of 10 CSF samples. The number of tumor cells counted in 7 of the samples ranged 
Table I. Clinical characteristics of 6 female patients with adenocarcinoma.

\begin{tabular}{|c|c|c|c|c|}
\hline Patient & Age, years & $\begin{array}{l}\text { Gene mutation, } \\
\text { EGFR/K-RAS }\end{array}$ & Metastatic parts & Major clinical manifestations \\
\hline 1 & 64 & $+/-$ & Meninx, brain & Headache, deaf, cauda equina syndrome \\
\hline 2 & 64 & Unknown & Meninx, brain & Cognitive disorder, dysopia \\
\hline 3 & 63 & $+/-$ & Meninx, brain & Headache, cauda equina syndrome \\
\hline 4 & 52 & $+/-$ & Meninx & Headache, deaf, cognitive disorder \\
\hline 5 & 52 & $-/-$ & Meninx, brain & Headache, dysopia \\
\hline 6 & 46 & $+/-$ & Meninx, brain & Headache \\
\hline
\end{tabular}

+, present; -, absent; EGFR, epidermal growth factor receptor; K-RAS, Kirsten rat sarcoma viral oncogene homolog.

Table II. Results of the analysis of 10 CSF samples from 6 patients, all with visible signs of meningeal metastasis on enhanced magnetic resonance imaging of the brain.

\begin{tabular}{lccc}
\hline Sample & $\begin{array}{c}\text { CSF TM-iFISH detection, } \\
\text { number of cells/7.5 ml CSF }\end{array}$ & $\begin{array}{c}\text { CSF tumor } \\
\text { cytological examination }\end{array}$ & $\begin{array}{c}\text { CSF protein } \\
\text { determination, } \mathrm{g} / \mathrm{I}^{*}\end{array}$ \\
\hline $1-1$ & 720 & Identifiable tumor cells & 0.72 \\
$1-2$ & 13 & Unseen tumor cells & 0.51 \\
$1-3$ & 12 & Unseen tumor cells & 0.62 \\
$2-1$ & 0 & Unseen tumor cells & 0.77 \\
$2-2$ & 0 & Unseen tumor cells & 0.55 \\
3 & 3 & Unseen tumor cells & 0.67 \\
$4-1$ & 1,832 & Identifiable tumor cells & 0.46 \\
$4-2$ & 246 & Unseen tumor cells & 0.43 \\
5 & 0 & Unseen tumor cells & 0.35 \\
6 & 1,133 & Identifiable tumor cells & 0.53 \\
\hline
\end{tabular}

"The normal level of CSF protein was 0.15-0.35 g/l. CSF, cerebrospinal fluid; TM-iFISH, tumor marker-immunostaining fluorescence in situ hybridization.

between 3-1,823 cells/7.5 ml CSF (Fig. 1). Tumor cells were indicated, but not counted, in 3 samples using CSF cytological examination. The albumin protein levels were increased compared with the normal range $(0.15-0.35 \mathrm{~g} / \mathrm{l})$ in 9 samples, when a CSF biochemical examination was performed. Additionally, TM-iFISH was used again to count the CSF malignant tumor cells in 3 patients following intrathecal injection chemotherapy of $10 \mathrm{mg}$ methotrexate and $5 \mathrm{mg}$ dexamethasone, once per week. The results indicated that the malignant tumor cell count of 2 patients was decreased compared with pre-treatment counts (Table II). The tumor cells in 1 patient were not successfully enriched and counted. The results of the brain enhanced MRI scan performed on all patients prior to and subsequent to treatment revealed signs of meningeal metastasis, but no significant changes (Table II).

\section{Discussion}

LM affects the brain tissue, cranial nerves and spinal cord and is caused by the metastasis and diffuse infiltration of tumor cells in primary lesions to the leptomeninges and subarachnoid space (1). Early diagnosis and treatment may effectively delay the neurological impairment caused by the progression of the pathological condition (2). At present, the following criteria are primarily used to diagnose LM: i) Definite tumor history; ii) the appearance of newly-onset neurological symptoms and signs; iii) typical MRI manifestations; and iv) tumor cells indicated using CSF cytological examinations (10). LM may be diagnosed if the patient demonstrates the first two criteria, plus one other.

The clinical manifestations of LM are frequently complicated, varied and non-specific due to different invasive regions of tumor cells; therefore, identifying the symptoms caused by metastasis of the brain parenchyma and spinal cord and the adverse reactions induced by the treatment of primary tumors may be challenging (2). The major clinical manifestations of LM include: i) Brain parenchyma involvement and meningeal irritation syndromes, including headaches, vomiting, nuchal rigidity, meningeal irritation, a change in mental status, clouding of the consciousness, cognitive disorders, the onset of symptomatic epilepsy and limb activity disorder; ii) symptoms of cranial nerve involvement, of which the optic, oculomotor, trochlear, abducens, facial and auditory nerves are frequently involved, including impaired vision, diplopia, facial numbness, 
gustatory and auditory abnormality, dysphagia and dysphonia; and iii) irritation symptoms of the spinal cord and spinal nerve roots, including segmental anesthesia, limb numbness, sensory ataxia, a weakening and disappearance of the tendon reflex, sphincter of Oddi dysfunction and radiculalgia (11). In the present study, the major clinical manifestations of the LM in the 6 patients were a persistent headache (5 patients), cognitive disorder ( 3 patients), dysopia ( 2 patients), deafness ( 2 patients) and cauda equina syndrome ( 2 patients), which conformed to the major clinical manifestations of LM. In addition, there were 5 patients that experienced complications of brain metastasis, and all patients were administered with intrathecal or intravenous chemotherapy. Therefore, the aforementioned symptoms are extremely challenging to identify and diagnose in the clinic.

The results of CSF biochemical examinations are abnormal in almost all patients with LM, but lack specificity (11). One of the major manifestation of LM is increased albumin protein levels, and the primary mechanisms include increased protein exudation as a result of the infiltration of tumor cells into the meninges, the chemical stimulation of tumor metabolites, damage to the blood brain barrier and increased vascular permeability. In the present study, $9(90 \%)$ of the CSF biochemical examinations revealed an increased albumin protein content (11). This indicates that CSF biochemical examination has an increased sensitivity compared with cytological examination, but lacks specificity. Therefore, it may only be used as an index for clinical application. The rate of patients demonstrating LM-negative results at the first CSF cytological examination is $\geq 45 \%$, and the rate of patients testing positive at the second examination is $\geq 80 \%$ (5). However, the detection rate may not be increased by $>3$ additional examinations (5). The specificity of enhanced MRI scans may reach $100 \%$ (12). The typical manifestations of LM include meningeal thickening, nodules accompanying the meninges, linear or stripe-like meninges, diffuse reinforcement, a dural tail sign, and secondary changes, such as a smaller capacity of the brain parenchyma, encephaledema and periventricular edema (12). However, $65 \%$ of scans falsely assess patients as LM-negative and $10 \%$ of scans falsely assess patients as LM-positive (12). In the present study, 6 patients were diagnosed with LM of lung cancer using a CSF cell smear or brain enhanced MRI scan, but tumor cells were only identified in 3 of the CSF cell smear samples, indicating that CSF cytological examination may provide random results and be an ineffective method to count tumor cells. The typical signs of LM were indicated in 6 patients subsequent to treatment on a brain enhanced MRI scan reexamination; however, the leptomeningeal metastatic range was not indicated to have changed conspicuously. Therefore, it is a challenge to evaluate efficacy using enhanced MRI scans. The authors of the present study suggest that CSF cytological examinations and enhanced MRI scans are unable to meet the standards of clinicians and efficacy evaluations in the diagnosis of LM. Therefore, it is essential to seek a more sensitive detection method.

Using enrichment and analysis technologies, TM-iFISH may effectively identify a variety of non-hematogenous tumor epithelial cells in biological fluid samples and demonstrate an increased sensitivity and specificity for the identification of CTCs (9). Certain studies indicate that TM-iFISH demonstrates an increased sensitivity and specificity in the diagnosis of breast cancer, malignant melanoma and lung cancer LM through the detection of CTCs in the CSF $(5,13)$. In the present study, 10 CSF samples from 6 patients were detected via TM-iFISH. In $7(70 \%)$ of the samples, the number of tumor cells identified ranged between 3 and 1,823 cells/7.5 ml CSF. TM-iFISH was used again to count the number of CSF malignant tumor cells in 3 patients subsequent to treatment, and the results indicated that the tumor cell counts of 2 of the patients decreased in comparison to pre-treatment counts. The tumor cells in 1 patient were not enriched and counted prior to and subsequent to treatment. This suggests that TM-iFISH may be a better cell counting tool in comparison to CSF cytological examinations and enhanced MRI scans, due to an increased sensitivity to CSF tumor cells, which may provide certain advantages in the diagnosis, efficacy evaluation and treatment of LM. Additionally, the findings of the present study revealed that TM-iFISH detected a markedly greater cell count in the 3 samples that contained identifiable tumor cells in the CSF cytological examinations, compared with the other 7 samples. Additional studies are required in order to determine whether there is an association between the tumor cells that were identifiable in the CSF cytological examination and the high number of cells counted using CSF TM-iFISH. Due to the small sample size, a benign brain group was not set up as a control in order to calculate the specificity of the TM-iFISH in the present study; however, the results may provide a reference for the design of future clinical studies.

\section{Acknowledgements}

The present study was supported by Tianjin municipal health bureau funded projects of science and technology (grant no. $2014 \mathrm{kz0} 42)$.

\section{References}

1. Leal T, Chang JE, Mehta M and Robins HI: Leptomeningeal metastasis: Challenges in diagnosis and treatment. Curr Cancer Ther Rev 7: 319-327, 2011.

2. Nagpal S, Riess J and Wakelee H: Treatment of leptomeningeal spread of NSCLC: A continuing challenge. Curr Treat Options Oncol 13: 491-504, 2012.

3. Liu ZH, Zhang DL and Wang L: The value of cerebrospinal fluid cytological checkup in diagnosing meningeal carcinomatosis. Zhonghua Quan Ke Yi Xue 8: 187-188, 2010.

4. Wang P, She CH, Li P, Pu YZ, Wang XG and Li WL: Significance of tumor markers in the diagnosis of lung cancer meningeal metastasis. Zhongguo Zhong Liu Lin Chuang 35: 61-64, 2008.

5. Le Rhun E, Massin F, Tu Q, Bonneterre J, De Carvalho Bittencourt M and Faure GC: Development of a new method for identification and quantification in cerebrospinal fluid of malignant cells from breast carcinoma leptomeningeal metastasis. BMC Clin Pathol 12: 21, 2012.

6. Igawa S, Gohda K, Fukui T, Ryuge S, Otani S, Masago A, Sato J, Murakami K, Maki S, Katono K, et al : Circulating tumor cells as a prognostic factor in patients with small cell lung cancer. Oncol Lett 7: 1469-1473, 2014.

7. Yu Y, Chen Z, Dong J, Wei P, Hu R, Zhou C, Sun N, Luo M, Yang W, Yao R, et al: Folate receptor-positive circulating tumor cells as a novel diagnostic biomarker in non-small cell lung cancer. Transl Oncol 6: 697-702, 2013.

8. Romiti A, Raffa S, Di Rocco R, Roberto M, Milano A, Zullo A, Leone L, Ranieri D, Mazzetta F, Medda E, et al: Circulating tumor cells count predicts survival in colorectal cancer patients. J Gastrointestin Liver Dis 23: 279-284, 2014. 
9. Jiang R, Ma CH, Zhu ZL, Li JD, Wang B, Sun LW and Lv Y: Application of circulating tumor cell detection in cerebrospinal fluid in the diagnosis of meningeal metastasis from non-small cell lung cancer. Zhong Guo Xiandai Shenjig Jibing Zazhi 14: 698-701, 2014.

10. Wang Y, Gao Y, Zhu YF and Tao RJ: The diagnosis and treatments progress of meningeal carcinomatosis. Zhongguo Lin Chuang Shen Jing Wai Ke Za Zhi 18: 760-762, 2013.

11. Ma C, Jiang R, Li J, Wang B, Sun L and Lv Y: Research progress of lung cancer with leptomeningeal metastasis. Zhongguo Fei A Za Zhi 17: 695-700, 2014 (In Chinese).
12. Clarke JL, Perez HR, Jacks LM, Panageas KS and Deangelis LM: Leptomeningeal metastases in the MRI era. Neurology 74: $1449-1454,2010$

13. Le Rhun E, Tu Q, De Carvalho Bittencourt M, Farre I, Mortier L, Cai H, Kohler C and Faure GC: Detection and quantification of CSF malignant cells by the CellSearch technology in patients with melanoma leptomeningeal metastasis. Med Oncol 30: 538, 2013. 